\title{
A single-center experience of steroid therapy for COVID-19 management
}

\author{
Aamir Shaukat ${ }^{1}$, Ahmad Ayaz Sabri ${ }^{2}$, Umair Ahmed ${ }^{1}$, Nazir Ahmad ${ }^{2}$, Dilshad \\ Muhammad $^{2}$, Majid $\mathrm{Ali}^{3}$, and Arfan Mahmood ${ }^{4}$
}

${ }^{1}$ Allied Hospital

${ }^{2}$ Faisalabad Medical University

${ }^{3} \mathrm{Umm}$ Al-Qura University

${ }^{4}$ Government General Hospital

January 11, 2021

\begin{abstract}
Aim: There is an emerging role of steroids in the management of COVID-19. We aimed to compare the outcome of COVID-19 patients (recovery versus mortality) who were treated with steroids with those who were not treated with steroids during their course of hospital stay. Methods: A retrospective analysis of all moderately to severely ill COVID-19 patients, meeting the inclusion and exclusion criteria, admitted to our center during the study period of four months, was performed. The patients were categorized into two groups: Group I included 25 patients who were given steroids, and Group II also included 25 patients who were not given any steroids during their hospital stay. The primary outcome (recovery versus mortality), length of hospital stay as well as other features were compared between the two groups. Results: The mean length of hospital stay was 9.3 days in the steroids group and 10.9 days in the non-steroids group with a p value of 0.249 . None of the patients was shifted to a ventilator in either group. One patient in the steroids group (4\%) and two patients in the non-steroids group (8\%) needed to be put on high flow nasal cannula. One patient died in the steroids group with a recovery rate of $96 \%$, while two patients died in the non-steroids group with a recovery rate of $92 \%$ ( $\mathrm{p}$ value 0.552 ). Conclusion: Treatment with steroids in moderately to severely ill COVID-19 patients did not decrease the length of hospital stay or mortality in our study.
\end{abstract}

Manuscript category: Short report

Full title:

A single-center experience of steroid therapy for COVID-19 management

Short running title:

Steroid therapy for COVID-19

\section{Full names of the authors:}

Aamir Shaukat ${ }^{1,2}$, Ahmad Ayaz Sabri ${ }^{2,3}$, Umair Ahmad ${ }^{1,2}$, Nazir Ahmad ${ }^{2,3}$, Dilshad Muhammad ${ }^{2,3}$, Majid $\operatorname{Ali}^{4,5}$, Arfan Mahmood ${ }^{6,7}$

\section{Author's institutional affiliations:}

${ }^{1}$ Department of Medicine, Allied Hospital, Faisalabad, Pakistan

${ }^{2}$ Department of Medicine, Faisalabad Medical University, Faisalabad, Pakistan

${ }^{3}$ Department of Medicine, Government General Hospital, Faisalabad, Pakistan 
${ }^{4}$ College of Pharmacy, Umm Al-Qura University, Makkah, Saudi Arabia

${ }^{5}$ Faculty of Health and Medical Sciences, University of Adelaide, Adelaide, Australia

${ }^{6}$ Department of Gastroenterology, Government General Hospital, Faisalabad, Pakistan

${ }^{7}$ Department of Gastroenterology, Faisalabad Medical University, Faisalabad, Pakistan

\section{Corresponding author:}

Majid Ali

College of Pharmacy, Umm Al-Qura University

Abdia Campus, Taif Road, Makkah, Saudi Arabia

Phone: 00966544500676

Email: maaali@uqu.edu.sa; majid.ali@hotmail.com

\section{Author contributions:}

AS conceived, designed, and supervised the study. AM managed the data collection. NA conducted the data analysis. All the other authors (AAS, UA, DM and MA) contributed to the drafting and critical review of the manuscript. All authors approved the final draft of the manuscript.

\section{Disclosure statement:}

The authros declare that there are no conflicts of interest.

\section{Acknowledgment:}

The authors would like to acknowledge the efforts of all the doctors, nurses, and paramedics who helped in data collection.

\section{A Single-Center Experience of Steroid Therapy for COVID-19 Management}

\section{Abstract}

Aim: There is an emerging role of steroids in the management of COVID-19. We aimed to compare the outcome of COVID-19 patients (recovery versus mortality) who were treated with steroids with those who were not treated with steroids during their course of hospital stay.

Methods: A retrospective analysis of all moderately to severely ill COVID-19 patients, meeting the inclusion and exclusion criteria, admitted to our center during the study period of four months, was performed. The patients were categorized into two groups: Group I included 25 patients who were given steroids, and Group II also included 25 patients who were not given any steroids during their hospital stay. The primary outcome (recovery versus mortality), length of hospital stay as well as other features were compared between the two groups.

Results: The mean length of hospital stay was 9.3 days in the steroids group and 10.9 days in the nonsteroids group with a p value of 0.249 . None of the patients was shifted to a ventilator in either group. One patient in the steroids group (4\%) and two patients in the non-steroids group (8\%) needed to be put on high flow nasal cannula. One patient died in the steroids group with a recovery rate of $96 \%$, while two patients died in the non-steroids group with a recovery rate of $92 \%$ ( $\mathrm{p}$ value 0.552 ).

Conclusion: Treatment with steroids in moderately to severely ill COVID-19 patients did not decrease the length of hospital stay or mortality in our study.

Keywords: COVID-19; steroids; recovery; mortality; hospital stay.

What is already known about this subject? 
There is an emerging role of steroids use in the management of COVID-19 patients.

Evidence regarding the efficacy of steroids use in the management of COVID-19 patients is conflicting.

\section{What does this study contribute to the literature?}

Our study adds to the body of evidence that steroids use in moderately to severely ill COVID-19 patents does not reduce the mortality and length of hospital stay.

\section{INTRODUCTION}

According to the World Health Organization (WHO), viral diseases are emerging as a serious public health issue worldwide. In the last two decades, many viral epidemics such as severe acute respiratory syndrome coronavirus (SARS-CoV) disease from 2002 till 2003, and hemagglutinin type 1 and neuraminidase type 1 (H1N1) influenza in 2009, have been reported. Most recently, SARS-CoV2 was detected for the first time in Wuhan, China, in December 2019. On February 11, 2020, the WHO named this disease, coronavirus disease 2019 (COVID-19). This new virus is highly contagious and has rapidly spread globally. On January 30, 2020, the outbreak was declared a Public Health Emergency of International Concern (PHEIC) by the WHO. On March 11, 2020, as the number of COVID-19 cases outside China increased 13 times and more than 114 countries were involved, the WHO declared the COVID-19 a pandemic. ${ }^{1}$ To date (December 26, 2020), Pakistan has 469,482 confirmed cases of COVID-19 with a total death toll of 9816 . $^{2}$ The majority of the patients with COVID-19 have an uneventful recovery. However, approximately $19 \%$ of the patients suffer from a progressive worsening of the disease leading to severe pneumonia in $14 \%$ and critical pneumonia in $5 \%$ of the patients, which leads to acute respiratory distress syndrome (ARDS). ${ }^{3}$ Multiple therapeutic agents are being used and tried across the world for COVID-19 treatment and the management of its symptoms and complications. ${ }^{4}$

Immune-mediated response from the host of COVID-19 can lead to cytokine storm that leads to enormous vascular inflammation, disseminated coagulation, and shock, leading to multi-organ failure and death. This inflammatory pathophysiology has led to the use of corticosteroids in COVID-19 management. ${ }^{3}$ Since corticosteroids cause immune suppression by impairing the innate immunity, their use has largely been discouraged due to the fear of worsening of viral propagation. ${ }^{5}$ The majority of the earlier studies involving the use of steroids in SARS-CoV disease and the Middle East respiratory syndrome coronavirus (MERS-CoV) disease showed adverse outcomes. ${ }^{6,7}$ The WHO and the Center for Disease Control and Prevention (CDC) specifically advised against the use of corticosteroids in COVID-19 management earlier in the pandemic. ${ }^{3}$ The recent RECOVERY trial has shown significantly improved outcomes with dexamethasone, in severe COVID-19 patients who required oxygen therapy or were on a mechanical ventilator. ${ }^{8}$ Initially, we did not use steroids for COVID-19 management at our center as per the earlier WHO and CDC recommendations. However, subsequently, steroids became part of our COVID-19 management protocol based on the emerging evidence as well as our national clinical management guidelines for COVID-19 infection. ${ }^{2}$ In this study, we retrospectively compared the outcome of COVID-19 patients (recovery versus mortality) who were treated with steroids with those who were not treated with steroids during their course of hospital stay.

\section{METHODS}

This was a retrospective study conducted in the department of medicine at Government General Hospital, Faisalabad, Pakistan, over a period of four months from March 15, 2020 till July 15, 2020. During this period, all patients meeting the inclusion and exclusion criteria were enrolled in the study, using nonprobability purposive sampling. Inclusion criteria: all patients with age between 18 to 80 years, both genders, COVID-19 polymerase chain reaction (PCR) positive or radiologically COVID-19, moderate/severe critical respiratory disease. The disease severity of COVID-19 was based on our national clinical management guidelines for COVID-19 infection. ${ }^{2}$ Exclusion criteria: age less than 18 or more than 80 years, pregnant females, mild respiratory disease, received plasma transfusion, received tocilizumab or remdesivir. The enrolled patients were categorized into two groups. Group I included 25 patients who were given steroids during their hospital stay, oral or injectable (as per national clinical management guidelines). Group II also included 25 patients 
who were not given any steroids during their hospital stay. All patient medical charts were reviewed and data regarding age, gender, length of hospital stay, the severity of the condition at presentation, maximum oxygen requirement, the need for ventilatory support, laboratory findings, the dose of steroids used, and the outcome of the patients (recovery versus mortality) were recorded. The study received ethical approval from the Ethical Review Committee of Faisalabad Medical University.

\section{RESULTS}

The age of the 50 COVID-19 patients whose medical charts were retrospectively reviewed, ranged from 21 to 80 years, with the mean age of 57 years in the steroids group and 42 years in the non-steroids group. Overall, $62 \%$ were male and $36 \%$ were female patients. Co-morbidities among the patients of the steroids group were diabetes mellitus $16 \%$, hypertension $24 \%$, ischemic heart disease $20 \%$, smoking $12 \%$, and among that of the non-steroids group were diabetes mellitus $24 \%$, hypertension $24 \%$, ischemic heart disease $36 \%$, smoking $16 \%$. Lymphopenia was present in $72 \%$ of the patients in the steroids group and $64 \%$ of the patients in the non-steroids group (see Table 1).

All patients in the steroids group received dexamethasone $6 \mathrm{mg}$ intravenously for one week as part of the protocol for COVID-19 management. The mean length of hospital stay was 9.3 days in the steroids group and 10.9 days in the non-steroids group with a p value of 0.249 (see Table 2). In the steroids group, $64 \%$ had moderate respiratory disease and $36 \%$ had severe respiratory disease. In the non-steroids group, $64 \%$ had moderate respiratory disease, $32 \%$ had severe respiratory disease and $4 \%$ had a critical disease. None of the patients was shifted to a ventilator in either group. One patient in the steroids group (4\%) and two patients in the non-steroids group $(8 \%)$ needed to be put on high flow nasal cannula. In the steroids group, $64 \%$ of patients and in the non-steroids group, $72 \%$ of patients had a maximum oxygen requirement of 1-5 $\mathrm{L} / \mathrm{min}$. One patient died in the steroids group with a recovery rate of $96 \%$, while two patients died in the non-steroids group with a recovery rate of $92 \%$ ( $\mathrm{p}$ value 0.552 ) (see Table 3 ).

\section{DISCUSSION}

The emerging role of steroids in the management of moderately to severely ill COVID-19 patients was the motivation for our study. This retrospective study was conducted to assess the role of steroids in the management of patients with moderate or severe COVID-19 infection. The outcome of patients, whether recovery from or death due to COVID-19 infection, who received steroids was compared with the patients who did not receive steroids as part of their treatment for COVID-19 infection. The other main parameter that was compared between the two groups was their length of hospital stay during the course of their illness, which may have been affected by the baseline comorbidities they might have. The majority of the patients in the steroids group $(96 \%)$ recovered uneventfully and were discharged from the hospital. The mortality rate in this group was $4 \%$ compared to that of $8 \%$ in the non-steroids group. Although the mortality rate was higher in the non-steroids group, it was statistically non-significant. Our findings are in line with a meta-analysis published in May 2020, which showed that corticosteroids did not significantly reduce the risk of death in COVID-19 patients. ${ }^{9}$ The mean length of hospital stay in our study was also less in the steroids group than that in the non-steroids group; however, it was non-significant. This is contrary to the results of the meta-analysis which showed that the steroids use increased the length of hospital stay. ${ }^{9}$ Severity of the disease among the two groups as well as the maximum oxygen requirement was found to be similar, which may indicate that the steroids had a minimal effect on the severity of the COVID-19 infection in our study.

Regarding the baseline comorbidities, lymphopenia was higher in the steroids group whereas the incidences of diabetes mellitus and ischemic heart disease were lower in this group. Whether this difference in the baseline comorbidities had any impact on our findings, we could not perform the regression analysis due to the limited sample size in order to address this issue. Additionally, since this was a retrospective analysis of the data of COVID-19 patients, we were unable to compare the other characteristics such as age and disease severity between the two groups. These limitations warrant larger multicenter randomized clinical trials with adequate sample size to compare the safety and efficacy of steroids in patients with moderate and severe COVID-19 infection and the factors affecting them. Moreover, since the steroids, as compared to 
antivirals, are inexpensive and readily available in developing countries, findings of these trials conducted in developing countries can provide enormous benefits and save a larger number of patients from complications with an economic impact on the limited resources.

\section{CONCLUSION}

Our single-center study concludes that treatment with steroids in moderately to severely ill COVID-19 patients did not decrease the length of hospital stay or mortality. However, larger multicenter randomized control trials are needed to determine the efficacy of steroids in reducing hospital stay and/or mortality in patients with moderate and severe COVID-19.

\section{REFERENCES}

1. Cascella M, Rajnik M, Cuomo A, et al. Features, evaluation, and treatment of coronavirus. [Updated 2020 Oct 4]. In: StatPearls [Internet]. Treasure Island (FL): StatPearls Publishing; January 2020.

2. Ministry of National Health Services Regulations and Coordination. COVID-19 health advisory platform. 2020. http://covid.gov.pk/. Accessed 26 December, 2020.

3. Singh A, Majumdar S, Singh R, Misra A. Role of corticosteroid in the management of COVID-19: a systemic review and a clinician's perspective. Diabetes \& Metabolic Syndrome: Clinical Research \& Reviews. 2020;14(5):971-978. doi:10.1016/j.dsx.2020.06.054

4. Tobaiqy M, Qashqary M, Al-Dahery $\mathrm{S}$ et al. Therapeutic management of patients with COVID-19: a systematic review. Infection Prevention in Practice. 2020;2(3):100061. doi:10.1016/j.infpip.2020.100061

5. Isidori A, Arnaldi G, Boscaro M et al. COVID-19 infection and glucocorticoids: update from the Italian Society of Endocrinology expert opinion on steroid replacement in adrenal insufficiency. J Endocrinol Invest. 2020;43(8):1141-1147. doi:10.1007/s40618-020-01266-w

6. Stockman L, Bellamy R, Garner P. SARS: systematic review of treatment effects. PLoS Med. 2006;3(9):e343. doi:10.1371/journal.pmed.0030343

7. Arabi Y, Mandourah Y, Al-Hameed F et al. Corticosteroid therapy for critically ill patients with Middle East respiratory syndrome. Am J Respir Crit Care Med. 2018;197(6):757-767. doi:10.1164/rccm.201706-1172oc

8. Horby P, Lim W, Emberson J et al. Effect of dexamethasone in hospitalized patients with COVID-19: preliminary report. 2020. doi:10.1101/2020.06.22.20137273 [Preprint]

9. Li H, Chen C, Hu F, et al. Impact of corticosteroid therapy on outcomes of persons with SARSCoV-2, SARS-CoV, or MERS-CoV infection: a systematic review and meta-analysis. Leukemia. 2020;34(6):1503-1511. doi:10.1038/s41375-020-0848-3

TABLE 1 Characteristics and comorbidities of the two groups

\begin{tabular}{|c|c|c|c|c|}
\hline $\begin{array}{l}\text { Characteristics/ } \\
\text { Comorbidities }\end{array}$ & $\begin{array}{l}\text { Group I (steroids } \\
\text { group) }(\mathrm{n}=\mathbf{2 5})\end{array}$ & $\begin{array}{l}\text { Group I (steroids } \\
\text { group) }(\mathrm{n}=25)\end{array}$ & $\begin{array}{l}\text { Group II } \\
\text { (non-steroids } \\
\text { group) }(n=25)\end{array}$ & $\begin{array}{l}\text { Group II } \\
\text { (non-steroids } \\
\text { group) }(n=25)\end{array}$ \\
\hline Gender & $72 \%$ male & $28 \%$ female & $56 \%$ male & $44 \%$ female \\
\hline Mean age & 57 years & 57 years & 43 years & 43 years \\
\hline Smokers & $12 \%$ & $12 \%$ & $16 \%$ & $16 \%$ \\
\hline $\begin{array}{l}\text { Moderate } \\
\text { respiratory } \\
\text { disease }\end{array}$ & $64 \%$ & $64 \%$ & $64 \%$ & $64 \%$ \\
\hline $\begin{array}{l}\text { Severe respiratory } \\
\text { disease }\end{array}$ & $36 \%$ & $36 \%$ & $32 \%$ & $32 \%$ \\
\hline $\begin{array}{l}\text { Critical } \\
\text { respiratory } \\
\text { disease }\end{array}$ & $0 \%$ & $0 \%$ & $4 \%$ & $4 \%$ \\
\hline Diabetes mellitus & $16 \%$ & $16 \%$ & $24 \%$ & $24 \%$ \\
\hline
\end{tabular}




\begin{tabular}{|c|c|c|c|c|}
\hline $\begin{array}{l}\text { Characteristics/ } \\
\text { Comorbidities }\end{array}$ & $\begin{array}{l}\text { Group I (steroids } \\
\text { group) }(\mathrm{n}=\mathbf{2 5})\end{array}$ & $\begin{array}{l}\text { Group I (steroids } \\
\text { group) }(\mathrm{n}=25)\end{array}$ & $\begin{array}{l}\text { Group II } \\
\text { (non-steroids } \\
\text { group) }(n=25)\end{array}$ & $\begin{array}{l}\text { Group II } \\
\text { (non-steroids } \\
\text { group) }(\mathbf{n}=\mathbf{2 5})\end{array}$ \\
\hline Hypertension & $24 \%$ & $24 \%$ & $24 \%$ & $24 \%$ \\
\hline $\begin{array}{l}\text { Ischemic heart } \\
\text { disease }\end{array}$ & $20 \%$ & $20 \%$ & $36 \%$ & $36 \%$ \\
\hline Lymphopenia & $72 \%$ & $72 \%$ & $64 \%$ & $64 \%$ \\
\hline
\end{tabular}

TABLE 2 Comparison of length of hospital stay between the two groups

\begin{tabular}{llll}
\hline Length of hospital stay & Groups & Groups & p value* \\
\hline & Group I (n=25) & Group II (n=25) & \\
Mean number of days (SD) & $9.32(4.53)$ & $10.96(5.37)$ & 0.249 \\
\hline
\end{tabular}

SD: Standard deviation

*Mann-Whitney U test

TABLE 3 Comparison of steroid treatment outcome between the two groups

\begin{tabular}{llll}
\hline Outcome & Groups & Groups & p value* \\
\hline & Group I $(\mathbf{n = 2 5 )}$ & Group II $(\mathbf{n}=\mathbf{2 5})$ & \\
Death (n) & $1(4 \%)$ & $2(8 \%)$ & 0.552 \\
Recovery (n) & $24(96 \%)$ & $23(92 \%)$ & \\
\hline
\end{tabular}

*Chi-square test 